\title{
The Silicon Strip Tracker of the Fermi Large Area Telescope
}

\author{
J. Bregeon*, on behalf of the Fermi-LAT collaboration ${ }^{\dagger}$ \\ Istituto Nazionale di Fisica Nucleare, \\ Largo Pontecorvo 3, 56127 Pisa, Italy \\ E-mail: johan.bregeon@pi.infn.it
}

The Large Area Telescope (LAT) is the primary instrument on-board the Fermi Gamma-ray Space Telescope (Fermi ), an observatory on a low Earth orbit that was launched on June $11^{\text {th }} 2008$ to monitor the high energy $\gamma$-ray sky. The LAT tracker is a solid-state instrument: tungsten foils convert the gamma rays into electron-positron pairs which are then tracked in silicon planes in order to reconstruct the incoming photon direction. The tracker comprises 36 planes of singlesided silicon strip detectors, for a total of 73 square meters of silicon, read out by nearly 900,000 amplifier-discriminator channels. The system operates on only $160 \mathrm{~W}$ of conditioned power while achieving $>99 \%$ single-plane efficiency within its active area and better than 1 channel per million noise occupancy. We describe the tracker design and performance, and discuss in particular the excellent stability of the hardware response during the first three years of operation on orbit.

The 2011 Europhysics Conference on High Energy Physics-HEP 2011,

July 21-27, 2011

Grenoble, Rhône-Alpes France

\footnotetext{
${ }^{*}$ Speaker.

$\dagger$ The Fermi LAT Collaboration acknowledges support from a number of agencies and institutes for both development and the operation of the LAT as well as scientific data analysis. These include NASA and DOE in the United States, CEA/Irfu and IN2P3/CNRS in France, ASI and INFN in Italy, MEXT, KEK, and JAXA in Japan, and the K. A. Wallenberg Foundation, the Swedish Research Council and the National Space Board in Sweden. Additional support from INAF in Italy for science analysis during the operations phase is also gratefully acknowledged.
} 
The Large Area Telescope (LAT) on-board the NASA Fermi Gamma-ray space telescope is a pair-conversion $\gamma$-ray detector able to measure $\gamma$-ray photons from $20 \mathrm{MeV}$ up to over $300 \mathrm{GeV}$ [1] . The LAT is built in a modular array of $4 \times 4$ identical towers, each of which consists of a silicon tracker/converter, a CsI hodoscopic calorimeter and an electronic module. The 16 tracker modules are covered by the Anti-Coincidence Detector (ACD). The $\gamma$-ray photon converts into a $e^{+} / e^{-}$pair in a tungsten foil of the tracker, and the $e^{+}$and $e^{-}$leave a track in the position-sensitive detectors below giving access to the direction of the original $\gamma$-ray in the sky. The $e^{+} / e^{-}$pairs then develop an electromagnetic shower in the calorimeter, from which the energy is reconstructed. This paper will first describe the design and construction of the LAT tracker and will then focus on the on-orbit behavior during the first 3 years of operations.

\section{Design and performances}

The basic brick of the tracker is a composite panel, or "tray", that provides the required mechanical and thermal qualities relying on a carbon-composite assembly and a vented aluminum honeycomb core. Each tray supports two silicon planes that are glued on each side. The tungsten foil radiator lies just above the bottom silicon plane. One single tracker tower is a pile of $18 \mathrm{x}-\mathrm{y}$ planes providing a high level of compactness, and dead areas reduced to a minimum ; only $2 \mathrm{~mm}$ separate 2 consecutive $\mathrm{x}-\mathrm{y}$ silicon planes and the space between towers is just $2 \mathrm{~mm}$.

Each silicon plane is made of 4 parallel ladders, each of which is built by connecting the strips of 4 Silicon Strip Detectors (SSD). We used $400 \mu \mathrm{m}$ thick single sided SSD of area $8.95 \times 8.95$ $\mathrm{cm}^{2}$, read by $384 \mathrm{Al}$ strips for a pitch of $228 \mu \mathrm{m}$. The silicon plane readout is done through 24 front-end chips and 2 digital readout controllers. Readout controllers may be configured to read from 0 to 24 front-end chips for redundancy, data can be shift either left or right which helps to mitigate the consequence of any dead chip. The digital signal is then brought to the main tower electronic module via flat flex cables: 2 cables on each of the 4 sides of the tower. The whole electronic chain has a very low power consumption, $200 \mu \mathrm{W}$ per channel, or an envelope of just $160 \mathrm{~W}$ for the whole tracker.

Overall, 18 complete tracker towers were built and tested in 9 months: 16 of them were chosen to be part of the main instrument and the remaining 2 were kept as spare. All the towers are well within the specifications with an efficiency always greater than $98 \%$ and a number of bad channels below $0.5 \%$ [2]..

\section{Hardware monitoring on-orbit}

All the relevant tracker quantities are thoroughly monitored on a run by run basis at all the levels of the data processing chain. For this review, we selected all the runs taken in the nominal data taking configuration and with good statistics: $~ 17000$ runs from September 2008 to June 2011. The strip efficiency is monitored for each strip by selecting minimum ionizing protons straight tracks, and looking for hits around the interpolated or extrapolated position using the two nearest hits associated with the track within the active area. Figure 1 shows the hit efficiency trending against time for the past $\sim 3$ years for tower 15 , a typical tracker tower. The averaged hit efficiency is at all time high and very stable, no hint of degradation is observed. 


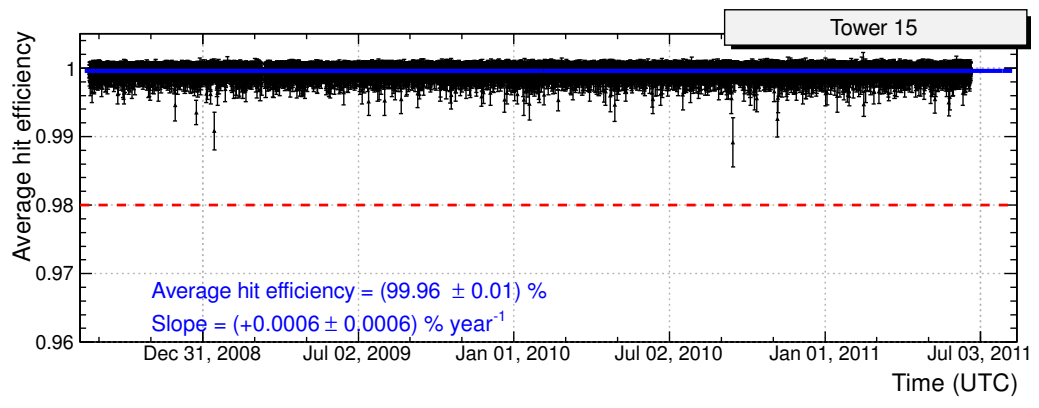

Figure 1: Time trending on a run by run basis of the hit efficiency in the tracker tower 15 .

The noise occupancy is monitored for each strip by measuring the accumulating strip hits on the silicon layers that are far from the triggering towers, and is additionally cross-checked by studying periodic triggers. As shown on top of figure 2 for one plane of tower 15, the noise occupancy is low and stable, at the level of $4 \times 10^{-3}$ for a layer of 1536 strips: requirements limit the noise occupancy to $8 \%$ for each layer. When a noisy strip is detected, the on-board hardware configuration is updated to mask it at the trigger level: only 416 strips out of almost a million $(<0.05 \%)$ are masked after 3 years on-orbit.

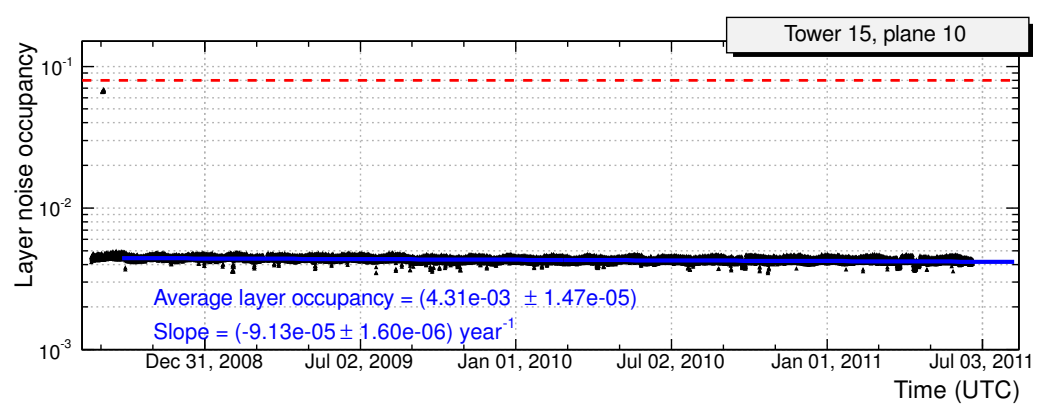

Figure 2: The noise occupancy for a typical tracker plane.

The time-over-threshold is monitored by using minimum ionizing proton tracks associated to a single hit in a tracker plane, and is corrected for its angular dependence. Since few months after launch, when the hardware configuration and calibration constants have been updated, the analog signal has been very stable with a drift slope smaller than one percent per year.

After 3 years of smooth operations on-orbit, characterized by a $>99 \%$ up-time, the LAT tracker is still performing extremely well and we have so far no indication of any potential serious hardware failure or premature aging of the detectors.

\section{References}

[1] Atwood et al., W. B., "The Large Area Telescope on the Fermi Gamma-Ray Space Telescope Mission," Astrophys. J. 697, 1071-1102 (June 2009).

[2] Atwood et al., W. B., "Design and initial tests of the tracker-converter of the Gamma-ray Large Area Space Telescope,” Astropart. Phys. 28, 422-434 (Dec. 2007). 